\title{
Total Intracranial Migration of Ventriculoperitoneal Shunt: A Case Report with Literature Review
}

\author{
Ahtesham Khizar ${ }^{1}$, Syed Aamir Shah ${ }^{1}$, Soha Zahid², and Jayant Yadav ${ }^{3}$ \\ ${ }^{1}$ Pakistan Institute of Medical Sciences \\ ${ }^{2}$ Jinnah Sindh Medical University \\ ${ }^{3}$ Annapurna Neurological Institute and Allied Sciences
}

March 3, 2022

\begin{abstract}
Total intracranial shunt migration is an extremely rare complication following ventriculoperitoneal shunting and it occurs probably due to raised intra-peritoneal pressure, strong head movements, and inadequate shunt fixation. The best possible approach to prevent shunt migration would be better patient handling along with appropriate operative techniques.
\end{abstract}

Total Intracranial Migration of Ventriculoperitoneal Shunt: A Case Report with Literature Review

Ahtesham Khizar ${ }^{1}$, Syed Aamir Shah ${ }^{1}$, Soha Zahid ${ }^{2}$, Jayant Kumar Yadav ${ }^{3}$

$1=$ Pakistan Institute of Medical Sciences, Islamabad, Pakistan

$2=$ Jinnah Medical and Dental College, Karachi, Pakistan

3 = Annapurna Neurological Institute and Allied Sciences, Kathmandu, Nepal

\section{Corresponding Author:}

Jayant Kumar Yadav, MBBS

Email: jkyadav22@iom.edu.np

Annapurna Neurological Institute and Allied Sciences, Kathmandu 44700, Nepal

\section{Abstract:}

Total intracranial shunt migration is an extremely rare complication following ventriculoperitoneal shunting and it occurs probably due to raised intra-peritoneal pressure, strong head movements, and inadequate shunt fixation. The best possible approach to prevent shunt migration would be better patient handling along with appropriate operative techniques.

\section{Key clinical message:}

Total intracranial shunt migration is a rare complication following ventriculoperitoneal shunt. Better anchorage of the shunt to the pericranium and peritoneum, optimum creation of subgaleal space and a smaller burr hole opening may be useful.

Key words: Intracranial migration, Ventriculoperitoneal shunt, Shunt complication.

\section{Introduction:}


Ventriculoperitoneal (VP) shunt is one of the most frequently performed procedures in neurosurgical practice. The first surgical procedure of VP shunt in the long-term treatment of hydrocephalus was performed by Kausch in 1908 (1). It is commonly used to treat different types of hydrocephalus like obstructive hydrocephalus and Normal Pressure Hydrocephalus (NPH). Shunt failure is the most common complication that occurs in $40-70 \%$ of the cases. Other complications may include shunt blockage, infection, over drainage, underdrainage and visual field defects. VP shunt migration is one of the common complications and can occur in both cranial and caudal direction. It can possibly migrate to other parts of the body such as gallbladder, anus, liver, umbilicus, colon and thorax. However, complete intracranial migration of VP shunt is a rare occurrence (. We herein present a case of a 10-month-old child who presented with complete intracranial migration of a VP shunt.

\section{Case presentation:}

A 10-month-old boy presented to our university hospital with the history of progressive enlargement of head, multiple episodes of vomiting and decreased oral intake for 3 days. He had Congenital Hydrocephalus and underwent medium pressure VP Shunt (Chhabra Shunt) at the age of one month at some other hospital. Following VP Shunt surgery, he remained well until this visit. On clinical examination, child appeared drowsy. He had an enlarged head with tense and bulging anterior fontanelle. Scalp veins were engorged and prominent. Sunset sign was present. Shunt chamber could not be palpated. Shunt failure was suspected based on history and clinical examination and X-ray was ordered. X-Ray shunt series showed an abnormal cranial migration of VP shunt assembly inside the cranium (Fig.1, A\&B). Computed Tomography (CT) brain Plain showed grossly dilated ventricles, subdural hygroma and VP shunt assembly inside the ventricles (Fig.2 A\&B). Routine investigations were within normal limits. At surgery, the previous burr hole site was explored. Burr hole was about $1 \mathrm{x} 1 \mathrm{~cm}$ in size. A rigid pediatric neuro endoscope was introduced through the burr hole and the entire shunt was seen coiled up inside the ventricle. It was removed with the help of an endoscopic grasper. A new VP shunt (Medtronic Shunt) was inserted on the opposite side. He had good postoperative recovery, improved clinically and was discharged in three days.

\section{Discussion:}

Total intracranial migration of VP shunt is an extremely rare complication. The incidence of total migration of VP shunt is reported to be about $0.1-0.5 \%$ (. The migration can take place in either cranial or caudal directions. Commonly, it is usually the distal or peritoneal catheter that migrates after disconnecting into many locations such as the scalp, mouth, thorax, diaphragm, heart, pulmonary artery, chest, breast, stomach, gall bladder, liver, scrotum, colon, bladder, vagina and rectum and causing injury to the local organs . However, upward migration has been reported as well. After a thorough review of literature, we found twenty-seven cases up to date of total intracranial VP shunt migration (Table 1).

Etiology of this complication is unknown, but several factors are thought to be responsible in facilitating proximal shunt migration. It is believed that the direction of shunt migration is dependent upon the pressure gradients between cranial and peritoneal cavities. Furthermore, it is hypothesized that pediatric patients are at an increased risk due to the shorter distance between the peritoneum and cranium. This short distance and violent, uncontrolled movements of the head (windlass effect) could lead to mechanical displacement of the shunt in cranial direction (. Another possible mechanism of proximal shunt migration may be due to inadequate fixation of shunt at the distal end or detachment due to an increased rate of growth in early stages of life. In addition, thin cerebral mantle, large ventricles and wide fontanelles create an intracranial pressure close to the atmospheric pressure while abdominal pressure remains positive in comparison to the atmosphere. This creates a pressure gradient which can lead to sucking of the shunt towards cranium . Interestingly, many other case studies report patient-related factors such as malnutrition (less subcutaneous tissue for anchorage), anemia, young age, thin cortical layer, and severe hydrocephalus which could all be related to upward shunt migration. Some authors propose factors like seizures, and constipation as responsible for possible migration (. In most VP shunt insertions, a burr hole is made in the occiput providing a straight path up from the peritoneum . Although this may be the easiest mode of insertion, it may contribute to cranial shunt migration as the path is simply too straightforward. Finally, in resource poor settings like ours 
"Chhabra" shunt is used as it is very economical for patients. However, it has cylindrical chamber which can easily slide into the cranium (. The best approach would be a frontal burr hole instead of occipital burr hole. Further, optimum creation of subgaleal space, the firm anchoring of connector sites to the periosteum while avoiding the large dural opening and large burr holes can help prevent such complications (2).

\section{Conclusion:}

Total intracranial migration of VP shunt is a rare but serious complication in patients with severe hydrocephalus. The best possible approach to prevent shunt migration would be better anchorage of the shunt to the pericranium and peritoneum, a frontal burr hole instead of occipital and a smaller burr hole opening. In addition, regular follow-up and educating parents regarding red flags and complications should be practiced readily.
Abbreviations:
CT $=$ Computed Tomography
NPH $=$ Normal Pressure Hydrocephalus
$\mathbf{V P}=$ Ventriculoperitoneal

\section{Declarations:}

Funding : No funding was required for the publication of this case report.

\section{Acknowledgement: None}

Conflicts of interest : None of the authors has any conflict of interest to disclose

Ethics of statement: Not applicable

Written consent from the patient: The patient's parents provided written informed consent for publication of this case report and accompanying images.

Detailed author's contribution: AK, SZ and JKY wrote the manuscript. AK and SAS were involved in the management of the patient. All authors read and approved the final manuscript.

Data availability statement: Not applicable

\section{References:}

\begin{tabular}{llll}
\hline S.N. & Author's Name & Year reported & No. of cases reported \\
1 & Mori K & 1975 & 1 \\
2 & Garijo JA & 1979 & 1 \\
3 & Villarejo F & 1979 & 1 \\
4 & Drigo G & 1983 & 2 \\
5 & Young HA (6 & 1983 & 2 \\
6 & Eljamel MS & 1995 & 1 \\
7 & Ahmed Ammar & 1995 & 1 \\
8 & Abou el Nasr HT & 1998 & 1 \\
9 & Gupta PK & 1999 & 1 \\
10 & C J Dominguez & 2000 & 1 \\
11 & Acharya R & 2002 & 1 \\
2 & Shimizu & 2002 & 1 \\
13 & C. Pereira & 2004 & 1 \\
14 & Nadkarni TD & 2007 & 1 \\
15 & Oluwole KE & 2007 & 1 \\
16 & Ali MN & 2008 & 1 \\
17 & Agarwal A & 2011 & 1
\end{tabular}




\begin{tabular}{llll}
18 & Shahram Shahsavaran & 2012 & 2 \\
19 & Vikas Naik & 2013 & 1 \\
20 & Arvind Malhotra & 2015 & 1 \\
21 & Rakesh Kumar Sharma & 2015 & 1 \\
22 & Elif Basaran Gundogdu & 2017 & 1 \\
23 & Rajendra Shrestha & 2018 & 1 \\
24 & Hammad Mehtab & 2021 & 1 \\
\hline
\end{tabular}

Table 1: Twenty-seven previously reported cases of intracranial migration of ventriculoperitoneal shunt in literature
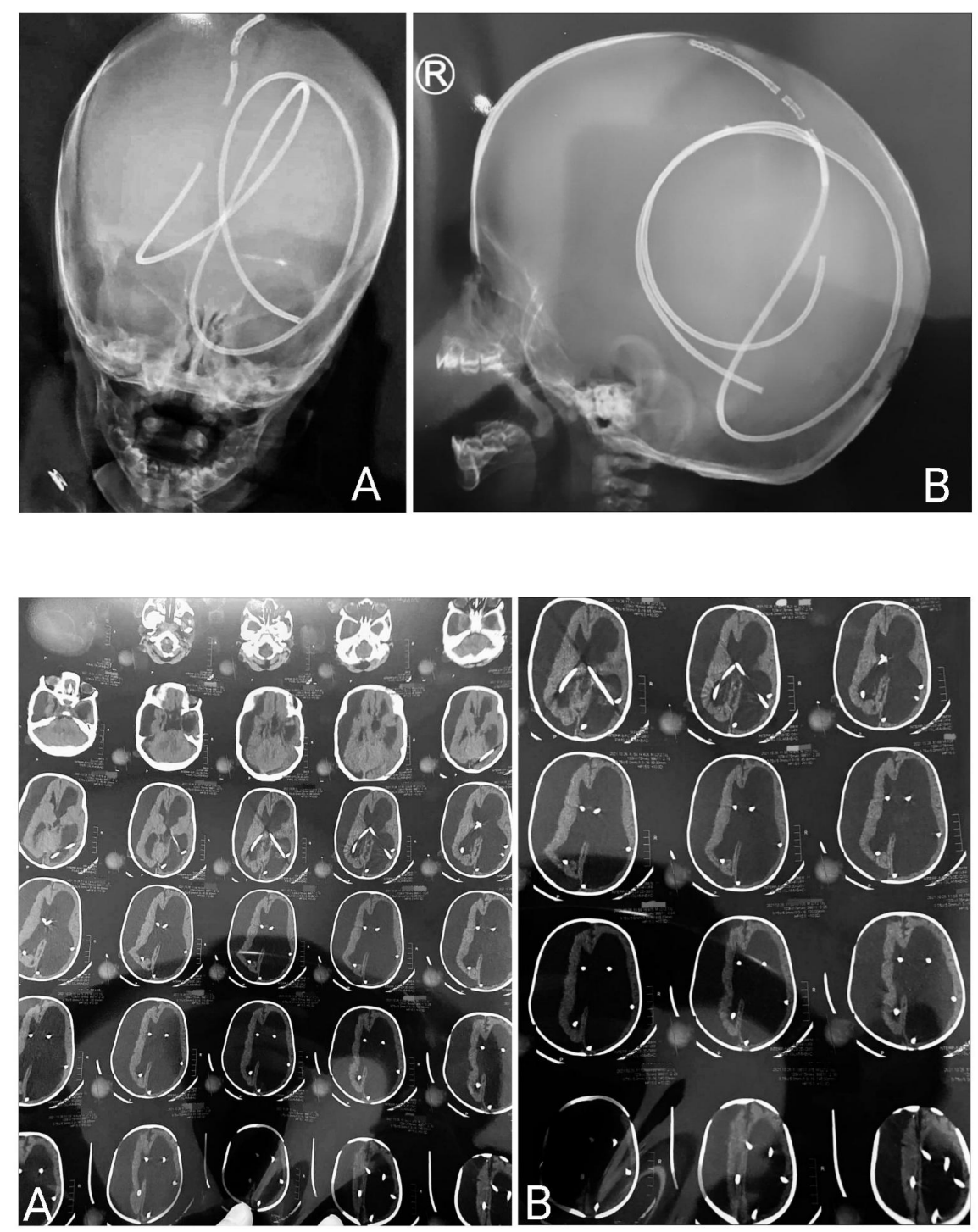